\title{
Conservation Characteristics and Nutritive Value of Sunflower Silages as Affected by The Maturity Stages and Fibrolytic Enzymes ${ }^{\#}$
}

\section{Sibel Erdoğan*, Murat Demirel \\ A R T I C L E I N F O \\ ${ }^{\#}$ The study is summarized from doctoral thesis of the first author. \\ Article history: \\ Received 11 January 2016 \\ Accepted 28 April 2016 \\ Available online, ISSN: 2148-127X}

Department of Animal Science, Faculty of Agriculture, Yuzuncu Yil University, 65080 Van, Turkey

Keywords:

Sunflower silage

Fermentation quality

Fibrolytic enzymes

Harvesting stage.

Yield properties

\section{A B S T R A C T}

Silage quality and nutritive value of sunflower silages ensiled with different level of fibrolytic enzymes at blooming, milk and dough stages were investigated. Fibrolytic enzyme complex (Viscozyme ${ }^{\circledR}$ L, V2010 Sigma Novozyme) was applied 0, 1, 1.5 and $2.5 \mathrm{ml} / \mathrm{kgDM}$ at ensiling. The dry matter (DM) yield increased with each increment of the maturity stage. Dry matter (DM), ether extract (EE) and crude cellulose (CC) contents of silages increased also in each delay in harvesting, while crude protein (CP), NDF and $\mathrm{ADF}$ contents of silages were the highest in blooming stage. The $\mathrm{pH}$ was higher in sunflower silage harvested at dough stage when compared to blooming stage, while acetic, propionic and butyric acid concentrations were all higher in blooming stage, although there were no differences in lactic acid concentrations among harvesting periods. Although silage structural carbohydrate composition was not significantly affected by any enzyme dose, Dose III enzyme treatment at the dough was associated with the highest concentration of lactic acid. Overall, there was no profound effect of enzyme supplementation on nutritive value and silage characteristics of sunflower silage.

${ }^{*}$ Corresponding Author:

E-mail: serdogan@yyu.edu.tr

\section{Introduction}

Sunflowers have a short production cycle, are resistant to cold, efficiently extract groundwater and are highly adaptable to different soil conditions, especially in comparison to other forage crops such as corn and sorghum (Gonçalves and Tomich, 1999; Tomich et al., 2003). The deep root system of the sunflower plant enables it to use approximately 92 percent of groundwater at a depth of $2 \mathrm{~m}$, compared to only 64 percent for the sorghum plant. Therefore sunflower has a viable option for producing forage sources for ruminants where a condition is not suitable for producing other forage crops. Alternative fodder silages are needed in places with and during periods of low rainfall or water (Bremner et al., 1986), and recent studies have focused on the use of sunflower seed husks (Marx, 1977), sunflower herbage (Lloveras, 1990), sunflower seed meal (Drackley et al., 1985) and sunflower silage (McGuffey and Schingoethe, 1980; Rodrigues et al., 2001; Hill et al., 2003; Bueno et al., 2004; Demirel et al., 2006; Rezende et al., 2007) in animal nutrition.

Harvesting stage is the most important affecting silage quality and digestibility. Dry matter content increases as harvest date is delayed, but as harvest date is delayed silage quality and digestibility which is the major factor effecting feed value declines. In order to obtain highquality sunflower silage, plants should be harvested during the last bloom, as early harvest results in losses due to high water content (Gregoire, 1999; Gonçalves et al., 1999). Ideally, ensiled sunflower should have a dry matter content of 30-40 percent; however, excessive delay in harvesting has the disadvantage of increasing fiber content.

The treatment of forage with fibrolytic enzymes has been proposed to improve fiber digestibility and to increase the amount of water-soluble carbohydrate (WSC) available as a substrate for lactic acid bacteria (LAB) (McDonald et al., 1991; Weinberg et al., 1995). Enzymes that are able to degrade cell wall components could add at ensiling to improve fermentation and animal performance (McDonald et al., 1991). In theory, degrading cell wall components to simpler molecules should provide silage bacteria with a more fermentable substrate (McDonald et al., 1991) and should increase the rate and extent of silage digestion in the rumen (Weinberg et al., 1995). Enzyme additives have been shown to improve fermentation characteristics in cases where limited substrate is able for fermentation (Fredeen and McQueen, 1993) and to reduce silage fiber content (Stokes, 1992).

This study aimed to identify the effect of different levels of fibrolytic enzyme added to sunflower herbage harvested at various stages on silage fermentation quality and dry matter yield. 


\section{Material and Methods}

The present study examined silages from green herbage sunflower harvested at blooming, milk and dough stages with the addition of Viscozyme ${ }^{\circledR}$ L (V2010 SigmaNovozyme), a commercially available multi-enzyme product comprised of cellulase, hemicelulase, xylanase and beta glucanase.

After soil preparation with different tillage machines, the sunflower seeds with $350 \mathrm{~g}$ for weight of 1000 seeds were sown by pneumatic seeder with row spacing of 70 $\mathrm{cm}$ and a tractor with seed spacing $25 \mathrm{~cm}$. During sowing $8 \mathrm{~kg} \mathrm{~N}$ and $8 \mathrm{~kg} \mathrm{P}$ fertilizer were given per decare. $8 \mathrm{~kg} \mathrm{~N}$ fertilizer was given after hoeing.

In each harvest stage, average $5 \mathrm{~m}^{2}$ area was harvested. In order to eliminate row edge effect one row was discarded. Crops obtained from each row were immediately weighed. Ten plants from each row were chosen to determine their plant, leaf, table and stalk weight and plant height. Samples taken from harvested plants were chopped and dried $\left(60^{\circ} \mathrm{C}\right)$ and dry matter yield calculated.

Green herbage of sunflower cut with silotrack at each stage and liquid fibrolytic enzyme complex added at ensiling, as follows: Blooming: Control 0 (no enzyme), Blooming-Dose I (1 ml/kgDM), Blooming-Dose II (1.5 $\mathrm{ml} / \mathrm{kgDM}$ ); Milk: Control 0 (no enzyme): Milk-Dose I (1 $\mathrm{ml} / \mathrm{kgDM})$, Milk-Dose II (1.5 ml/kgDM), Milk-Dose III (2.5ml/kgDM); Dough: Control 0 (no enzyme): DoughDose I (1 ml/kgDM), Dough-Dose II (1.5 ml/kgDM), Dough-Dose III (2.5 ml/kgDM). The chopped sunflowers were tightly filled in 9 plastic (120 1) barrels for each silage groups. Then, barrels were turned upside down and placed approximately $20 \mathrm{~cm}$ in the soil for incubation. Barrels were opened 90 days later.

Silage samples were taken from upper, central and bottom of each barrel. Silage $\mathrm{pH}$ was determined with a glass electrode after homogenization of $25 \mathrm{~g}$ of silage with $100 \mathrm{ml}$ of distilled water for $2 \mathrm{~min}$ in a blender (Hart and Horn, 1987). Then the remaining silage fluid was filtered through Whatman 54 paper, centrifuged and stored at $-20^{\circ} \mathrm{C}$. Lactic, acetic, propionic and butyric acids in silage fluids were analyzed using gas chromatography (Madrid et al., 1999). All of silage samples were analyzed for dry matter (DM), crude protein $(\mathrm{CP})$, ether extract (EE), and ash (AOAC, 1990), acid detergent fiber (ADF) and neutral detergent fiber (NDF) (Van Soest and Robertson, 1979). PROC GLM in SAS/STAT (SAS,
2007) was used for all data analysis. Mean treatment differences were determined by Duncan's multiple range tests with a level of statistical differences of $5 \%$.

\section{Results and Discussion}

\section{Yield Properties}

Green herbage yields ( $\mathrm{kg} / \mathrm{da})$ of sunflower harvested at blooming, milk and dough stages were 3278.6, 7921.1 and $8166.3 \mathrm{~kg} / \mathrm{da}$, respectively. Green herbage yield increased when harvest was delayed from blooming to milk stage, but no significant increase was found between green herbage yield harvested at milk and dough stages ( $\mathrm{P}<0.05$; Table 1). But, dry matter yields increased linearly with delays in harvesting, from $466.9 \mathrm{~kg} / \mathrm{da}$ at blooming to $1693.5 \mathrm{~kg} / \mathrm{da}$ at milk and $1980.3 \mathrm{~kg} / \mathrm{da}$ at dough stages. Mean plant height at blooming was $2.3 \mathrm{~m}$, which was significantly lower than at milk $(2.7 \mathrm{~m})$ and dough $(2.7 \mathrm{~m})$ stages $(\mathrm{P}<0.05)$. Sunflower head ratios increased and leaf and stalk ratios decreased with maturity. Head ratio at blooming (18.1\%) was significantly lower when compared to milk (44.0\%) and dough $(37.7 \%)$ stages $(\mathrm{P}<0.05)$. Leaf ratio at blooming $(24.3 \%)$ was significantly higher than at milk (19.0\%) and dough $(18.79 \%)$ stages $(\mathrm{P}<0.05)$, and stalk ratio was also significantly higher at blooming $(57.6 \%)$ than at milk $(36.9 \%)$ and dough $(53.5 \%)$ stages $(\mathrm{P}<0.05)$.

The agronomic characteristics of sunflower green herbage harvested at different vegetation periods reported here are similar to those reported by earlier studies (Gonçalves and Tomich, 1999; Tomich et al., 2003; Demirel et al., 2006; Rezende et al., 2007). Green herbage and dry matter yields of different varieties ranged from $1280 \mathrm{~kg}$ to $2910 \mathrm{~kg} / \mathrm{da}$ and from $360 \mathrm{~kg}$ to $770 \mathrm{~kg} / \mathrm{da}$ respectively, whereas mean sunflower leaf, stalk and head ratios were reported as $19.7 \%, 33.7 \%$ and $46.7 \%$, respectively (Tomich et al., 2003).

\section{Nutrient Composition}

Dry matter contents of silages increased with a delay in harvesting, from $19.63 \%$ in silage harvested at blooming to $22.94 \%$ in silage harvested at milk and $31.34 \%$ in silage harvested at the dough stage (Table 2a, $2 \mathrm{~b} ; \mathrm{P}<0.05)$. Enzyme addition at blooming resulted in an increase in dry matter; however, similar effects on DM were not observed $(\mathrm{P}>0.05)$ at milk and dough stages. The highest DM content was obtained at dough stage $(\mathrm{P}<0.05)$.

Table 1 Yield properties of sunflower herbages harvested at different stages

\begin{tabular}{l|cccc}
\hline \multirow{2}{*}{ Variables* } & \multicolumn{3}{c}{ Harvesting Stages } \\
\cline { 2 - 5 } & $\mathrm{N}$ & Blooming Stage & Milking Stage & Dough Stage \\
\hline Herbage yield, kg/da & 3 & $3278.6 \pm 287.12^{\mathrm{b}}$ & $7921.1 \pm 287.12^{\mathrm{a}}$ & $8166.3 \pm 287.12^{\mathrm{a}}$ \\
Dry matter yield kg/da & 3 & $466.9 \pm 65.40^{\mathrm{c}}$ & $1693.5 \pm 65.40^{\mathrm{b}}$ & $1980.3 \pm 65.40^{\mathrm{a}}$ \\
Leaf ratio, \% & 3 & $24.3 \pm 1.37^{\mathrm{a}}$ & $19.0 \pm 1.37^{\mathrm{b}}$ & $18.8 \pm 1.37^{\mathrm{b}}$ \\
Head ratio, \% & 3 & $18.1 \pm 0.80^{\mathrm{c}}$ & $44.0 \pm 0.80^{\mathrm{a}}$ & $37.7 \pm 0.80^{\mathrm{b}}$ \\
Stalk ratio, \% & 3 & $57.6 \pm 1.34^{\mathrm{a}}$ & $36.9 \pm 1.34^{\mathrm{c}}$ & $53.5 \pm 1.34^{\mathrm{b}}$ \\
Plant height, $\mathrm{m}$ & 3 & $2.3 \pm 0.08^{\mathrm{b}}$ & $2.7 \pm 0.08^{\mathrm{a}}$ & $2.7 \pm 0.08^{\mathrm{a}}$ \\
\hline
\end{tabular}

$\mathrm{a}, \mathrm{b}, \mathrm{c}$ : Values with different superscripts in the same line differ signficantly $(\mathrm{P}<0.05)$. 
Table 2a Nutrient composition of sunflower silage with different amounts of fibrolytic enzyme supplements and harvested at different stages $(\%)$

\begin{tabular}{|c|c|c|c|c|c|c|}
\hline \multicolumn{2}{|c|}{ Enzyme Doses } & $\mathrm{n}$ & DM & $\mathrm{OM}$ & $\mathrm{CA}$ & $\mathrm{EE}$ \\
\hline \multicolumn{2}{|c|}{ Harvesting stage } & & $* *$ & $\mathrm{NS}$ & $\mathrm{NS}$ & $* *$ \\
\hline \multicolumn{2}{|c|}{ Blooming } & 9 & $19.6 \pm 0.51^{\mathrm{c}}$ & $86.4 \pm 0.17$ & $7.8 \pm 0.14$ & $5.6 \pm 0.23^{3}$ \\
\hline \multicolumn{2}{|l|}{ Milking } & 12 & $22.9 \pm 0.45^{\mathrm{b}}$ & $85.9 \pm 0.15$ & $7.8 \pm 0.12$ & $13.0 \pm 0.20^{2}$ \\
\hline \multicolumn{2}{|l|}{ Dough stage } & 12 & $31.3 \pm 0.45^{\mathrm{a}}$ & $86.2 \pm 0.15$ & $7.5 \pm 0.12$ & $15.2 \pm 0.20^{1}$ \\
\hline \multicolumn{2}{|l|}{ Doses } & & & & $*$ & \\
\hline \multicolumn{2}{|c|}{ Control } & 9 & $23.0 \pm 1.74$ & $86.0 \pm 0.17$ & $7.8 \pm 0.13^{\mathrm{a}}$ & $11.8 \pm 1.36$ \\
\hline \multicolumn{2}{|c|}{ Dose I(1ml/kgKM) } & 9 & $25.4 \pm 1.74$ & $86.3 \pm 0.17$ & $7.7 \pm 0.13^{\mathrm{ab}}$ & $10.8 \pm 1.36$ \\
\hline \multicolumn{2}{|c|}{ Dose II (1.5ml/kgKM) } & 9 & $25.3 \pm 1.74$ & $86.4 \pm 0.17$ & $7.3 \pm 0.13^{\mathrm{b}}$ & $11.4 \pm 1.36$ \\
\hline \multicolumn{2}{|c|}{ Dose III (2.5ml/kgKM) } & 6 & $27.3 \pm 2.13$ & $86.1 \pm 0.21$ & $7.9 \pm 0.16^{\mathrm{a}}$ & $14.0 \pm 1.66$ \\
\hline \multicolumn{2}{|c|}{ Harvesting stagexdose } & & $*$ & $* *$ & $* *$ & $*$ \\
\hline \multirow{3}{*}{ Blooming } & Control & 3 & $17.3 \pm 0.51 \mathrm{E}^{\mathrm{b}}$ & $86.1 \pm 0.12 \mathrm{CDEF}^{\mathrm{b}}$ & $8.0 . \pm 0.17 \mathrm{AB}$ & $5.5 \pm 0.18 \mathrm{G}^{\mathrm{ab}}$ \\
\hline & Dose I & 3 & $20.6 \pm 0.51 \mathrm{D}^{\mathrm{a}}$ & $86.8 \pm 0.12 \mathrm{AB}^{\mathrm{a}}$ & $7.9 \pm 0.17 \mathrm{AB}$ & $5.2 \pm 0.18 \mathrm{G}^{\mathrm{b}}$ \\
\hline & Dose II & 3 & $21.0 \pm 0.51 \mathrm{D}^{\mathrm{a}}$ & $86.3 \pm 0.12 \mathrm{BCDE}^{\mathrm{b}}$ & $7.5 \pm 0.17 \mathrm{BC}$ & $6.0 \pm 0.18 \mathrm{G}^{\mathrm{a}}$ \\
\hline \multirow{4}{*}{ Milking } & Control & 3 & $21.7 \pm 0.72 \mathrm{CD}$ & $85.3 \pm 0.22 \mathrm{G}^{\mathrm{b}}$ & $7.8 \pm 0.21 \mathrm{AB}^{\mathrm{a}}$ & $13.6 \pm 0.46 \mathrm{DE}$ \\
\hline & Dose I & 3 & $23.3 \pm 0.72 \mathrm{C}$ & $85.6 \pm 0.22 \mathrm{FG}^{\mathrm{b}}$ & $8.0 \pm 0.21 \mathrm{AB}^{\mathrm{a}}$ & $12.9 \pm 0.46 \mathrm{EF}$ \\
\hline & Dose II & 3 & $23.5 \pm 0.72 \mathrm{C}$ & $86.9 \pm 0.22 \mathrm{~A}^{\mathrm{a}}$ & $6.9 \pm 0.21 \mathrm{D}^{\mathrm{b}}$ & $12.5 \pm 0.46 \mathrm{~F}$ \\
\hline & Dose III & 3 & $23.3 \pm 0.72 \mathrm{C}$ & $86.0 \pm 0.22 \mathrm{DEF}^{\mathrm{b}}$ & $8.2 \pm 0.21 \mathrm{~A}^{\mathrm{a}}$ & $13.2 \pm 0.46 \mathrm{EF}$ \\
\hline \multirow{4}{*}{ Dough Stage } & Control & 3 & $30.1 \pm 0.75 \mathrm{~B}$ & $86.5 \pm 0.14 \mathrm{ABCD}^{\mathrm{a}}$ & $7.7 \pm 0.10 \mathrm{ABC}^{\mathrm{a}}$ & $16.1 \pm 0.21 \mathrm{~A}^{\mathrm{a}}$ \\
\hline & Dose I & 3 & $32.4 \pm 0.75 \mathrm{~A}$ & $86.5 \pm 0.14 \mathrm{ABC}^{\mathrm{a}}$ & $7.2 \pm 0.10 \mathrm{CD}^{\mathrm{b}}$ & $14.3 \pm 0.21 \mathrm{CD}^{\mathrm{c}}$ \\
\hline & Dose II & 3 & $31.5 \pm 0.75 \mathrm{AB}$ & $85.8 \pm 0.14 \mathrm{EFG}^{\mathrm{b}}$ & $7.5 \pm 0.10 \mathrm{BC}^{\mathrm{ab}}$ & $15.56 \pm 0.21 \mathrm{AB}^{\mathrm{ab}}$ \\
\hline & Dose III & 3 & $31.4 \pm 0.75 \mathrm{AB}$ & $86.2 \pm 0.14 \mathrm{CDEF}^{\mathrm{ab}}$ & $7.6 \pm 0.10 \mathrm{BC}^{\mathrm{a}}$ & $14.9 \pm 0.21 \mathrm{BC}^{\mathrm{bc}}$ \\
\hline
\end{tabular}

$* * \mathrm{P}<0.01 ; * \mathrm{P}<0.05 ;{ }^{\mathrm{a}, \mathrm{b}, \mathrm{c}}$ :The values with different number between harvesting periods, enzyme doses and doses of each harvesting periods in the same column differ significantly $(\mathrm{P}<0.05)$; A, B, C, D, E, F, G: The values with different superscripts in the same column differ significantly $(\mathrm{P}<0.05)$.

Table $2 \mathrm{~b}$ Nutrient composition of sunflower silage with different amounts of fibrolytic enzyme supplements and harvested at different stages $(\%)$

\begin{tabular}{|c|c|c|c|c|c|c|}
\hline \multicolumn{2}{|c|}{ Enzyme Doses } & $\mathrm{n}$ & $\mathrm{CP}$ & $\mathrm{CC}$ & NDF & ADF \\
\hline \multicolumn{2}{|c|}{ Harvesting stage } & & $*$ & $* *$ & $*$ & $* *$ \\
\hline \multicolumn{2}{|c|}{ Blooming } & 9 & $12.3 \pm 0.23^{\mathrm{a}}$ & $35.4 \pm 0.55^{\mathrm{c}}$ & $59.1 \pm 1.13^{\mathrm{a}}$ & $42.6 \pm 0.73^{\mathrm{a}}$ \\
\hline \multicolumn{2}{|l|}{ Milking } & 12 & $11.5 \pm 0.20^{\mathrm{b}}$ & $41.9 \pm 0.47^{\mathrm{b}}$ & $55.9 \pm 0.98^{\mathrm{b}}$ & $39.2 \pm 0.63^{\mathrm{b}}$ \\
\hline \multicolumn{2}{|l|}{ Dough stage } & 12 & $11.9 \pm 0.20^{\mathrm{ab}}$ & $44.9 \pm 0.47^{\mathrm{a}}$ & $56.5 \pm 0.98^{\mathrm{ab}}$ & $40.0 \pm 0.63^{\mathrm{b}}$ \\
\hline \multicolumn{2}{|l|}{ Doses } & & * & & & \\
\hline \multicolumn{2}{|c|}{ Control } & 9 & $12.2 \pm 0.23^{\mathrm{a}}$ & $41.5 \pm 1.39$ & $56.5 \pm 1.20$ & $39.9 \pm 0.85$ \\
\hline \multicolumn{2}{|c|}{ Dose I $(1 \mathrm{ml} / \mathrm{kgKM})$} & 9 & $11.4 \pm 0.23^{\mathrm{b}}$ & $40.5 \pm 1.39$ & $57.2 \pm 1.20$ & $40.7 \pm 0.85$ \\
\hline \multicolumn{2}{|c|}{ Dose II (1.5ml/kgKM) } & 9 & $11.9 \pm 0.23^{\mathrm{ab}}$ & $40.1 \pm 1.39$ & $58.2 \pm 1.20$ & $41.4 \pm 0.85$ \\
\hline \multicolumn{2}{|c|}{ Dose III (2.5ml/kgKM) } & 6 & $12.0 \pm 0.29^{\mathrm{ab}}$ & $43.7 \pm 1.70$ & $55.6 \pm 1.47$ & $39.5 \pm 1.04$ \\
\hline \multicolumn{2}{|c|}{ Harvesting stagexdose } & & $*$ & NS & NS & $*$ \\
\hline \multirow{3}{*}{ Blooming } & Control & 3 & $13.1 \pm 0.34 \mathrm{~A}^{\mathrm{a}}$ & $36.0 \pm 0.89 \mathrm{D}$ & $57.4 \pm 2.04 \mathrm{ABC}$ & $40.6 \pm 1.09 \mathrm{BCD}$ \\
\hline & Dose I & 3 & $11.8 \pm 0.34 \mathrm{BC}^{\mathrm{b}}$ & $34.5 \pm 0.89 \mathrm{D}$ & $60.9 \pm 2.04 \mathrm{~A}$ & $44.2 \pm 1.09 \mathrm{~A}$ \\
\hline & Dose II & 3 & $12.0 \pm 0.34 \mathrm{BC}^{\mathrm{ab}}$ & $35.7 \pm 0.89 \mathrm{D}$ & $59.0 \pm 2.04 \mathrm{ABC}$ & $42.9 \pm 1.09 \mathrm{AB}$ \\
\hline \multirow{4}{*}{ Milking } & Control & 3 & $11.9 \pm 0.17 \mathrm{BC}^{\mathrm{a}}$ & $42.5 \pm 0.96 \mathrm{BC}$ & $53.6 \pm 2.14 \mathrm{C}$ & $37.9 \pm 1.49 \mathrm{D}$ \\
\hline & Dose I & 3 & $11.2 \pm 0.17 \mathrm{C}^{\mathrm{b}}$ & $42.1 \pm 0.96 \mathrm{BC}$ & $54.4 \pm 2.14 \mathrm{BC}$ & $38.1 \pm 1.49 \mathrm{D}$ \\
\hline & Dose II & 3 & $11.5 \pm 0.17 \mathrm{BC}^{\mathrm{ab}}$ & $40.7 \pm 0.96 \mathrm{C}$ & $60.2 \pm 2.14 \mathrm{AB}$ & $42.2 \pm 1.49 \mathrm{ABC}$ \\
\hline & Dose III & 3 & $11.5 \pm 0.17 \mathrm{BC}^{\mathrm{ab}}$ & $42.2 \pm 0.96 \mathrm{BC}$ & $55.4 \pm 2.14 \mathrm{ABC}$ & $38.7 \pm 1.49 \mathrm{CD}$ \\
\hline \multirow{4}{*}{ Dough Stage } & Control & 3 & $11.7 \pm 0.43 \mathrm{BC}$ & $46.0 \pm 1.07 \mathrm{~A}$ & $58.5 \pm 1.38 \mathrm{ABC}$ & $41.0 \pm 0.52 \mathrm{ABCD}^{\mathrm{a}}$ \\
\hline & Dose I & 3 & $11.3 \pm 0.43 \mathrm{C}$ & $44.9 \pm 1.07 \mathrm{AB}$ & $56.2 \pm 1.38 \mathrm{ABC}$ & $39.7 \pm 0.52 \mathrm{BCD}^{\mathrm{ab}}$ \\
\hline & Dose II & 3 & $12.0 \pm 0.43 \mathrm{BC}$ & $43.7 \pm 1.07 \mathrm{ABC}$ & $55.5 \pm 1.38 \mathrm{ABC}$ & $39.0 \pm 0.52 \mathrm{CD}^{\mathrm{b}}$ \\
\hline & Dose III & 3 & $12.6 \pm 0.43 \mathrm{AB}$ & $45.2 \pm 1.07 \mathrm{AB}$ & $55.9 \pm 1.38 \mathrm{ABC}$ & $40.3 \pm 0.52 \mathrm{BCD}^{\mathrm{ab}}$ \\
\hline
\end{tabular}

The different nutrient composition of silage as affected by the forage type, amount and activity of enzyme added, and harvesting period was reported by the Harrison et al. (1994). Sneddon et al. (1981) found a DM content of $25.1 \%$ in sunflower silage harvested 120 days after sowing. Camara et al (1999a) reported that DM content of sunflower harvested at 5 different physiological stages $(65,81,94,108$ and 121 days) had a DM content of $10.9 \%, 14.7 \%, 16.1 \%, 22.5 \%$ and $35.1 \%$, respectively. Our values are similar to those reported in other studies (Valdez et al., 1986; Henrique et al., 1998; Rezende et al., 2001; Tomich et al., 2003; Rodrigues et al., 2005; Pereira et al., 2005; Ko et al., 2005).

Ether extract content of silages increased $(\mathrm{P}<0.05)$ from $5.6 \%$ at blooming to $13.0 \%$ at milk and to $15.2 \%$ dough stage, respectively. Application of enzyme level did not affect $(\mathrm{P}>0.05)$ ether extract ratios (Table 2a, 2b). Previous studies (Valdez et al., 1988; Henrique et al., 
1998) have also reported that ether extract content of sunflower increased gradually from blooming to dough stages. Camara and Monterio (1999) reported that ether extract contents of sunflower silages at blooming and maturity stages were $11.8 \%$ and $18.88 \%$, respectively. The ether extract content of sunflower silage in this study was similar to other studies (Hill et al., 2003; Demirel et al., 2006; Camara et al., 1999b).

Crude protein (CP) content of silages ensiled at blooming $(12.3 \%)$ was higher $(\mathrm{P}<0.05)$ than the $\mathrm{CP}$ levels at milk and dough stages. Although the addition of enzymes (Dose I) at dough stage had no effect on CP levels, CP was significantly lowered when enzymes were added (Dose I) at the blooming and milk stages $(\mathrm{P}<0.05)$ (Table 2a, 2b). In general, CP levels of silages were over than $10 \%$, which is similar to values reported by Sneddon et al. (1981), Tomich et al. (2004), Rezende et al. (2007) and Fassio et al. (2007).

Crude cellulose (CC) content of silages at blooming $(35.4 \%)$ was significantly lower $(\mathrm{P}<0.05)$ than the silages ensiled at milk (41.9\%) and dough (44.9\%) stages $(\mathrm{P}>0.05)$; the addition of enzymes did not affect $\mathrm{CC}$ levels at any maturity stage of ensiling (Table 2a, 2b). A previous study by Henrique et al. (1998) reported CC levels of silages with a range from $25.6 \%-26.3 \%$. Hill et al. (2003) found that $\mathrm{CC}$ content of sunflower silage increased with progressive maturity from the blooming to the dough period. Camara et al. (1999b) harvested sunflower at four different growth period $(56,68,94$ and 103 days) and reported increased $\mathrm{CC}$ content with maturity $(26.36 \%, 26.75 \%, 27.77 \%$ and $30.56 \%$, respectively) but found enzyme doses had no effect on CC levels.

The NDF content at blooming $(59.1 \%)$ was significantly higher than at milk $(55.9 \%)$ and dough $(56.5 \%)$ stages $(\mathrm{P}<0.05)$. The addition of enzymes did not affect NDF content of silages (Table 2a, 2b). The ADF content at blooming (42.6\%) was significantly higher than ADF content measured at milk $(39.2 \%)$ and dough $(40.0 \%)$ stages $(\mathrm{P}<0.05)$. Although the addition of enzymes at blooming and milk stages had no effect on ADF levels, ADF levels decreased when enzymes (dose II) were added at the dough stage $(\mathrm{P}<0.05$; Table 2a, 2b). Several studies suggest that cell-wall degrading enzymesfor example, cellulases can improve silage fermentation or alter the fiber content of silages (Kung et al., 1991; Almeida et al, 1995). These values are consistent with the findings in other studies (Valdez et al., 1998; Tomich et al., 2004; Y1ldiz et al., 2010) and enzyme doses did not affect the concentration of NDF and ADF (Gwayumba, 1997).

\section{Fermentation Quality}

Silage fermentation characteristics ( $\mathrm{pH}$, lactic acid, acetic acid, propionic acid, butyric acid) are given in Table 3. Silage $\mathrm{pH}$ values at blooming (4.1) and milk stages were similar (4.3), but the $\mathrm{pH}$ was significantly higher at the dough stage $(4.4) \quad(\mathrm{P}<0.05)$. Enzyme application at blooming and milk stages had no positive effect on silage $\mathrm{pH}$; however, $\mathrm{pH}$ was significantly lowered as a result of enzyme application at the dough stage $(\mathrm{P}<0.05)$, which had a positive effect on fermentation. Overall, the blooming stage control (3.9) and Dose II (4.0) silages had the lowest pH levels, whereas the dough stage control silage had the highest $\mathrm{pH}$ level $(\mathrm{P}<0.05)$ in line with the other studies (Schingoethe et al, 1980; Tomich et al., 2004; Pereira et al., 2005; Demirel et al., 2006, Mafakher et al., 2010). This may be explained by the higher lactic acid concentration and DM content of silages as reported by Rezende et al. (2007) who stated that fermentation was limited by the lower levels of water-soluble carbohydrates and higher dry matter contents of silages at the dough stage when compared to earlier stages of development.

Harvesting period was not found to have a significant effect on lactic acid concentrations. The highest concentration $(88.3 \mathrm{~g} / \mathrm{kg} \mathrm{DM})$ was observed at blooming. Enzyme supplements had varied effects on lactic acid concentrations. At the blooming stage, enzyme supplements had no positive effect on lactic acid concentrations, but negatively affected the LA concentration. However, at the milk stage, significant differences were found between Dose I $(96.6 \mathrm{~g} / \mathrm{kg} \mathrm{DM})$ and Dose II $(75.3 \mathrm{~g} / \mathrm{kg} \mathrm{DM})(\mathrm{P}<0.05)$, at the dough stage, Dose I and Dose II had no significant effect on lactic acid concentrations; however, the highest dose (Dose III) resulted in significantly higher lactic acid concentrations $(91.6 \mathrm{~g} / \mathrm{kg} \mathrm{DM})(\mathrm{P}<0.05)$. This resulted in a decrease in $\mathrm{pH}$ of silage at dough stage. Some earlier studies have reported the addition of fibrolytic enzymes to increase lactic acid concentrations (Zhu et al., 1999). In the present study, lactic acid levels were similar to those reported by Demirel et al. (2006), Pereira et al. (2005), Ko et al. (2005) and Tomich et al. (2004).

The acetic, propionic and butyric acid concentrations decreased with maturity $(\mathrm{P}<0.05)$. Acetic acid concentrations of sunflower silage decreased when harvesting was delayed from the blooming stage (36.0 $\mathrm{g} / \mathrm{kgDM})$ to the milk $(28.0 \mathrm{~g} / \mathrm{kgDM})$ and dough stages (23.5 g/kgDM) ( $\mathrm{P}<0.05$; Table 3). Enzyme supplements at the blooming and dough stages did not significantly affect acetic acid concentrations; however, at the milk stage, higher levels of supplements (Dose II) resulted in a significantly higher acetic acid when compared to lower levels of supplements (Dose I) $(\mathrm{P}<0.05)$. Propionic and butyric acids concentrations of sunflower silage decreased when harvesting was delayed from the blooming stage to the milk and dough stages $(\mathrm{P}<0.05$; Table 3$)$.

In conclusion, the dry matter yield of sunflower increased, but fiber content was similar with maturity. Moreover, increasing EE level with maturity suggests sunflowers could harvest at dough stage due to a similar silage fermentation patterns. 
Table 3 Fermentation quality of sunflower silage with different amounts of fibrolytic enzyme supplements and harvested at different stages

\begin{tabular}{|c|c|c|c|c|c|c|c|}
\hline \multicolumn{2}{|l|}{ Enzyme Doses } & $\mathrm{n}$ & $\mathrm{pH}$ & $\begin{array}{l}\text { Lactic Acid } \\
\text { g/kgDM }\end{array}$ & $\begin{array}{l}\text { Acetic Acid } \\
\text { g/kgDM }\end{array}$ & $\begin{array}{l}\text { Propionic Acid } \\
\text { g/kgDM }\end{array}$ & $\begin{array}{l}\text { Butyric Acid } \\
\text { g/kgDM }\end{array}$ \\
\hline \multicolumn{2}{|c|}{ Harvesting Stage } & & $* *$ & & $* *$ & $*$ & $*$ \\
\hline \multicolumn{2}{|c|}{ Blooming } & 9 & $4.1 \pm 0.07^{\mathrm{b}}$ & $88.3 \pm 4.54$ & $36.0 \pm 2.36^{1}$ & $21.0 \pm 1.17^{\mathrm{a}}$ & $1.0 \pm 0.25^{\mathrm{a}}$ \\
\hline \multicolumn{2}{|l|}{ Milking } & 12 & $4.3 \pm 0.06^{\mathrm{ab}}$ & $85.8 \pm 3.94$ & $28.0 \pm 2.05^{2}$ & $17.2 \pm 1.01^{\mathrm{b}}$ & $0.2 \pm 0.21^{\mathrm{b}}$ \\
\hline \multicolumn{2}{|l|}{ Dough Stage } & 12 & $4.4 \pm 0.06^{\mathrm{a}}$ & $76.7 \pm 3.94$ & $23.5 \pm 2.05^{2}$ & $17.0 \pm 1.01^{\mathrm{b}}$ & $0.2 \pm 0.21^{\mathrm{b}}$ \\
\hline \multicolumn{2}{|l|}{ Doses } & & $*$ & & & $*$ & \\
\hline \multicolumn{2}{|l|}{ Control } & 9 & $4.3 \pm 0.08^{\mathrm{ab}}$ & $86.2 \pm 4.62$ & $31.2 \pm 3.11$ & $16.3 \pm 1.14^{\mathrm{b}}$ & $0.4 \pm 0.27$ \\
\hline \multicolumn{2}{|c|}{ Dose I (1ml/kgKM) } & 9 & $4.3 \pm 0.08^{\mathrm{ab}}$ & $80.3 \pm 4.62$ & $29.2 \pm 3.11$ & $21.1 \pm 1.14^{\mathrm{a}}$ & $0.7 \pm 0.27$ \\
\hline \multicolumn{2}{|c|}{ Dose II (1.5ml/kgKM) } & 9 & $4.3 \pm 0.08^{b}$ & $77.7 \pm 4.62$ & $28.3 \pm 3.11$ & $16.6 \pm 1.14^{\mathrm{b}}$ & $0.3 \pm 0.27$ \\
\hline \multicolumn{2}{|c|}{ Dose III (2.5ml/kgKM) } & 6 & $4.5 \pm 0.10^{\mathrm{a}}$ & $91.4 \pm 5.66$ & $26.4 \pm 3.81$ & $18.7 \pm 1.40^{\mathrm{ab}}$ & $0.1 \pm 0.33$ \\
\hline \multicolumn{2}{|c|}{ Harvesting stagexdoses } & & $* *$ & $* *$ & $*$ & $* *$ & $*$ \\
\hline \multirow{3}{*}{ Blooming } & Control & 3 & $3.9 \pm 0.04 \mathrm{G}^{\mathrm{b}}$ & $104.4 \pm 9.05 \mathrm{~A}$ & $43.78 \pm 6.99 \mathrm{~A}$ & $16.7 \pm 1.33 \mathrm{~B}^{\mathrm{b}}$ & $0.6 \pm 0.77 \mathrm{~B}$ \\
\hline & Dose I & 3 & $4.5 \pm 0.04 \mathrm{BC}^{\mathrm{a}}$ & $73.2 \pm 9.05 \mathrm{CD}$ & $33.1 \pm 6.99 \mathrm{ABCD}$ & $27.7 \pm 1.33 \mathrm{~A}^{\mathrm{a}}$ & $2.0 \pm 0.77 \mathrm{~A}$ \\
\hline & Dose II & 3 & $4.0 \pm 0.04 \mathrm{G}^{\mathrm{b}}$ & $87.5 \pm 9.05 \mathrm{ABCD}$ & $37.1 \pm 6.99 \mathrm{AB}$ & $18.6 \pm 1.33 \mathrm{~B}^{\mathrm{b}}$ & $0.4 \pm 0.77 \mathrm{~B}$ \\
\hline \multirow{4}{*}{ Milking } & Control & 3 & $4.2 \pm 0.05 \mathrm{EF}^{\mathrm{b}}$ & $80.2 \pm 5.28 \mathrm{BCD}^{\mathrm{ab}}$ & $25.7 \pm 1.78 \mathrm{BCD}^{\mathrm{bc}}$ & $15.4 \pm 1.62 \mathrm{~B}$ & $0.4 \pm 0.09 \mathrm{~B}$ \\
\hline & Dose I & 3 & $4.3 \pm 0.05 \mathrm{DE}^{\mathrm{ab}}$ & $96.6 \pm 5.28 \mathrm{AB}^{\mathrm{a}}$ & $34.1 \pm 1.78 \mathrm{ABC}^{\mathrm{a}}$ & $18.8 \pm 1.62 \mathrm{~B}$ & $0.1 \pm 0.09 \mathrm{~B}$ \\
\hline & Dose II & 3 & $4.2 \pm 0.05 \mathrm{EF}^{\mathrm{b}}$ & $75.3 \pm 5.28 \mathrm{CD}^{\mathrm{b}}$ & $22.3 \pm 1.78 \mathrm{CD}^{\mathrm{c}}$ & $15.4 \pm 1.62 \mathrm{~B}$ & $0.3 \pm 0.09 \mathrm{~B}$ \\
\hline & Dose III & 3 & $4.4 \pm 0.05 \mathrm{BCD}^{\mathrm{a}}$ & $91.2 \pm 5.28 \mathrm{ABC}^{\mathrm{ab}}$ & $30 . \pm 1.78 \mathrm{BCD}^{\mathrm{ab}}$ & $19.2 \pm 1.62 \mathrm{~B}$ & $0.2 \pm 0.09 \mathrm{~B}$ \\
\hline \multirow{4}{*}{ Dough Stage } & Control & 3 & $4.7 \pm 0.06 \mathrm{~A}^{\mathrm{a}}$ & $73.9 \pm 2.84 \mathrm{CD}^{\mathrm{b}}$ & $24.2 \pm 2.06 \mathrm{BCD}$ & $16.7 \pm 0.92 \mathrm{~B}$ & $0.3 \pm 0.12 \mathrm{~B}$ \\
\hline & Dose I & 3 & $4.1 \pm 0.06 \mathrm{~F}^{\mathrm{c}}$ & $71.0 \pm 2.84 \mathrm{D}^{\mathrm{b}}$ & $20.4 \pm 2.06 \mathrm{D}$ & $16.8 \pm 0.92 \mathrm{~B}$ & $0.1 \pm 0.12 \mathrm{~B}$ \\
\hline & Dose II & 3 & $4.3 \pm 0.06 \mathrm{CDE}^{\mathrm{b}}$ & $70.4 \pm 2.84 \mathrm{D}^{\mathrm{b}}$ & $25.9 \pm 2.06 \mathrm{BCD}$ & $15.9 \pm 0.92 \mathrm{~B}$ & $0.2 \pm 0.12 \mathrm{~B}$ \\
\hline & Dose III & 3 & $4.5 \pm 0.06 \mathrm{~B}^{\mathrm{b}}$ & $91.6 \pm 2.84 \mathrm{ABC}^{\mathrm{a}}$ & $23.5 \pm 2.06 \mathrm{CD}$ & $18.2 \pm 0.92 \mathrm{~B}$ & $0.1 \pm 0.12 \mathrm{~B}$ \\
\hline
\end{tabular}

** $\mathrm{P}<0.01 ;{ }^{*} \mathrm{P}<0.05 ;{ }^{\mathrm{a}, \mathrm{b}, \mathrm{c}}$ :The values with different number between harvesting periods, enzyme doses and doses of each harvesting periods in the same column differ significantly $(\mathrm{P}<0.05)$. A, B, C, D, E, F, G: The values with different superscripts in the same column differ significantly $(\mathrm{P}<0.05)$

\section{Acknowledgments}

This study was supported by Yüzüncü Yıl University Scientific Research Project fund under the project number BBAP 2006 FBE D48. The abstract of this article was presented at VII National Animal Science Congress.

\section{References}

Almeida MF, Tiesenhausen IMEV, Aquino LH, Carvalho VD, Rocha GP, Silva, MGCM. 1995. Composição química e consumo voluntário das silagens de sorgo, em dois estádios de corte, girassol e milho para ruminantes. Ciência e Prática, 19(3): 315-321.

AOAC. 1990. Official methods of analysis. $15^{\text {th }}$ Edition, Association of Official Analytical Chemists, Arlington, Virginia, USA.

Bremner PM, Preston GK, Groth CF. 1986. A field comparison of sunflower (Helianthus annuus L.) and sorghum (Sorghum bicolor) in a long drying cycle. I. Water extraction. Australian J. Agric. Res., 37(5): 483-493.

Bueno MS, Junior EF, Possenti RA, Bianchini D, Leinz FF, Rodrigues CFC. 2004. Desempenho de cordeiros alimentados com silagem de girassol ou de milho com proporções crescentes de ração concentrada. Rev. Bras. Zootec., 33(6): 1942-1948.

Camara SMS, Monteiro CA. 1999. Desempenho vegetativo e reprodutivo de quatro cultivares de girassol (Helianthus annus L.) sob cinco densidades de plantas em época safrinha. XIII Reunião Nacional De Pesquisa De Girassol, Simpósio Nacional Sobre A Cultura De Girassol. Itumbiara. Anais., Londrina: Embrapa Soja, 121-122.

Camara GM, Silva SC, Monteiro CA, Mattiazzi P. 1999a. Determinação do momento ideal de colheita de girassol (Helianthus annuus L.) para ensilagem durante a safrinha de 1998. XIII Reunião Nacional De Pesquisa De Girassol. Itumbiara, Embrapa. 126-129.
Camara GM, Silva SC, Andrade FME, Monteiro CA, Mattiazzi P. 1999b. Determinação do momento ideal de colheita de girassol (Helianthus annuus L.) para ensilagem durante a safrinha de 1997. XIII Reunião Nacional De Pesquisa De Girassol. Itumbiara, Embrapa. 123-125.

Demirel M, Bolat D, Çelik S, Bakıcı Y, Çelik S. 2006. Quality of silages from sunflower harvested at different vegatational stages. J. Appl. Anim. Res., 30: 161-165.

Drackley JK, Clark AK, Sahlu T. 1985. Evaluation of sunflower crop residue in rations for growing Holstein heifers. J. Dairy Sci., 68: 2390-2395.

Fassio A, Gimenez A, Fernandez E, Martins DV, Cozzolino, D. 2007. Prediction of Chemical composition in sunflower whole plant and silage (Helianthus annus L.) by near infrared reflectance spectroscopy. J. Near Infrared Spectrosc., 15: 201207.

Fredeen AH, McQueen RE. 1993. Effects of enzyme additives on quality of alfalfa/grass silage and dairy cow performance. Can. J. Anim. Sci., 73: 581-585.

Gonçalves LC, Tomich TR. 1999. Utilização do girassol como silagem para alimentação bovina. Reunião Nacional De Pesquisa De Girassol, Itumbiara. Londrina:Embrapa Soja, 13: 21-30.

Gonçalves LC, Rodriguez NM, Pereira LGR, Rodrigues JAS, Borges I, Borges ALCC, Saliba EOS. 1999. Evaluation of different harvest times of four genotypes of sunflower (helianthus annuus 1.) for ensiling. FAO Electronic Conference on Tropical Silage. 1-6

Gregoire T. 1999. Sunflower silage. Available from http://www.ag.ndsu.nodak.edu/aginfo/procrop/sun/sunsil09.htm. (accessed February 10, 2005).

Gwayumba W. 1997. Lactic acid bacterial noculants and fibrolytic enzymes in forage preservation and degradability. (unpublished thesis) Phd. Diss. Saskatoon, University of Saskatchewan, 222

Harrison JH, Blauwiekel R, Stokes MR. 1994. Fermentation and utilization of grass silage. J. Dairy Sci., 77(10): 3209-3235. 
Hart SP, Horn FP. 1987. Ensiling characteristics and digestibility of combinations of turnips and wheat straw. J. Anim. Sci., 14: 1790-1800.

Henrique W, Andrade JB, Sampaio AAM. 1998 Silagem de milho, sorgo, girassol e suas comparações. II. Composição Bromatológica. Reunião Anual Da Sociedade Brasileira De Zootecnia, 35: 379-381.

Hill JAG, Flemming JS, Montanhini NR, Camargo H, Flemming DF. 2003. Valor nutricional do girassol (Helianthus annuus L.) como forrageira. Archives Vet. Sci., 8(1): 41-48.

Ko HJF, Reis RB, Gonçalves LC, Rodrigues JAS., Rodriguez NM, Sousa BM. 2005. Consumo voluntário e digestibilidade aparente da matéria seca, matéria orgânica, energia e parede celular das silagens de quatro genótipos de girassol (Helianthus annus). Arq. Bras. Med. Vet. Zootec., 57(5): 616-622.

Kung LJr, Tung RS, Maciorowski KG, Buffum K, Knutsen K. 1991. Effects of plant cell-wall-degrading enzymes and lactic acid bacteria on silage fermentation and composition. J. Dairy Sci., 74: 4284-4296.

Lloveras J. 1990. Dry matter yield and nutritive value of four summer annual crops in north-west Spain (Galicia). Grass Forage Sci., 45: 243-248.

Madrid J, Megjas MD, Hernandez F. 1999. Determination of short chain volatile fatty acids in silages from artichoke and orange by-products by capillary gas chromatography. J. Sci. Food Agric., 79: 580-584.

Mafakher E, Meskarbashee M, Hassibi P, Mashayekhi MR. 2010. Study of chemical composition and quality and charactersitics of corn, sunflower and corn-sunflower mixture silages. Asian Journal of Anim. and Vet. Adv., 5(2): 175-179.

Marx G.D. 1977. Utilization of sunflower silage, sunflower hulls with poultry litter and sunflower hulls mixed with corn silage for growing dairy animals. J. Dairy Sci., 60: 112-115.

McDonald P, Henderson AR, Heron SJE. 1991. The Biochemistry of Silage. Chalcombe Publication, Second Edition, UK. 340

McGuffey RK, Schingoethe DJ. 1980. Feeding value of high oil variety of sunflowers as silage to lactating dairy cows. J. Dairy Sci., 63 (7): 1109-1113.

Pereira LGR., Gonçalves LC, Tomich TR, Borges I, Rodriguez NM. 2005. Silos experimentais para avaliação da silagem de três genótipos de girassol (Helianthus annuus L.). Arq. Bras. Med. Vet. Zootec., 57(5): 690-696.

Rezende AV, Evangelista AR, Siqueira GR, Santos RV. 2001. Qualidade da silagem de girassol (Helianthus annuus L.). Diferentes idades de maturação fisiológica na safrinha. XXXVIII Reunião Anual Da Sociedade Brasileira De Zootecnia. Piracicaba. Anais...SBZ, 231-232.

Rezende AV, Evangelista AR, Valeriano AR, Siqueria GR, Vilela HH, Lope J. 2007. Valor nutritivo de silagens de seis cultivares de girassol em diferentes idades de corte. Ciênc Agrotec, Lavras, 31(3): 896-902.
Rodrigues PHM, Almeida MT, Melotti L, Andrade SJT, Júnior KCP. 2001. Efeitos da adição de inoculantes microbianos sobre a composição bromatológica e sobre a fermentação da silagem de girassol produzida em silos experimentais. Rev. Bras. Zootec., 30(6): 2169-2175.

Rodrigues PHM, Almeida TF, Meyer PM, Borgatti LMO. 2005. Valor nutritivo da silagem de girassol inoculada com bactérias ácido-láticas. Rev. Bras. Zootec., 34(1): 340-344.

SAS. 2007. SAS ${ }^{\circledR}$ User's Guide: Statistics, Version 8. SAS Institute Inc., Cary, NC.

Schingoethe DJ, Skyberg EW, Rook JA. 1980. Chemical composition of sunflower silage as influenced by additions of urea, dried whey and sodium hydroxide. J. Anim. Sci., 50: 625629.

Sneddon DN, Thomas VM, Roffler RE, Glen AM. 1981. Laboratory investigations of hydroxide-treated sunflower or alfalfa-grass silage. J. Anim. Sci., 53(6): 1623-1681.

Stokes MR. 1992. Effects of an enzyme mixture, an inoculant, and their interaction on silage fermentation and dairy production. J. Dairy Sci., 15: 764.

Tomich TR, Rodrigues JAS, Gonçalves LC, Tomich RGP, Carvalho AU. 2003. Potencial forrageiro de cultivares de girassol produzidos na safrinha para ensilagem. Arq. Bras. Med. Vet. Zootec., 55(6): 756-762.

Tomich TR, Gonçalves LC, Tomich RGP, Rodrigues JAS, Borges I, Rodrigues NM. 2004. Características químicas e disgestibilidde in vitro de silagens de girassol. Revista Brasileira de Zootecnia, 33(6): 1672-1682

Valdez FR, Fransen SC, Harrison JH. 1986. Corn-sunflower intercropping as a silage crop. J. Dairy Sci., 69, 138

Valdez FR, Harrisons JH, Deetz D, Frasen SC. 1988. In vivo digestibility of corn and sunflower intercropped as a silage crop. J. Dairy Sci., 71(7): 1860-1867.

Van Soest PJ, Robertson JB. 1979. Systems of analyses for evaluation of fibrous feed. In "Proc. Int. Workshop on Standardization of Analytical Methodology for Feeds". Ed, W. J. Pigden, C. C. Balch, and M. Graham. Int. Dev. Res. Center. Ottowa

Weinberg ZG, Ashbell G, Bolsen KK, Pahlow G, Hen Y, Azrieli A. 1995. The effect of a propionic acid bacterial inoculant applied at ensiling, with or without lactic acid bacteria, on the aerobic stability of pearl-millet and maize silages. J. Appl. Bacteriol., 78: 430-436.

Yildız C, Öztürk İ, Erkmen Y. 2010. Effects of chopping length and compaction values on the feed qualities of sunflower silage. Scientific Research and Essays, 5(15): 2051-2054.

Zhu Y, Naoki N, Kishida Y, Uchida S. 1999. Ensiling characteristics and ruminal degradation of Italian ryegrass and lucerne silages treated with cell wall-degrading enzymes. J. Sci. Food Agric., 79: 1987-1992. 